\title{
Diagnosing Hematological Disorders Using Deep Learning Method
}

\author{
(iD Tuba Karagül Yıldız ${ }^{1}$, iD Nilüfer Yurtay², (iD Birgül Öneç3 \\ ${ }^{1}$ Corresponding Author; Sakarya University, Institute of Natural Sciences, Computer and Information Sciences, \\ Turkey; tkaragul@sakarya.edu.tr; +90 5065187628 \\ ${ }^{2}$ Sakarya University, Department of Computer Engineering, Turkey; nyurtay@sakarya.edu.tr; \\ ${ }^{3}$ Duzce University, Medical School, Department of Internal Medicine, Turkey; birgulonec@gmail.com;
}

Received 5 December 2020; Revised 31 May 2021; Accepted 19 July 2021; Published online 31 August 2021

\begin{abstract}
Deciding on the diagnosis of the disease is an important step for treating the patients. Also, the numerical value of blood tests, the personal information of patients, and most importantly, an expert opinion is necessary to diagnose a disease. With the development of technology, patient-related data are obtained both rapidly and in large sizes. Deep learning methods, which can produce meaningful results by processing the data in raw form, are beginning to give results that are close to human opinion nowadays. The present work is aimed to develop a system that will enable the diagnosis of anemia in general practice conditions due to the increasing number of patients and the intention of the hospitals, as well as the difficulties in reaching the expert medical consultant. The main contribution of this work is to make a diagnosis like a doctor with the data as the way the doctor uses it. The data set was obtained from the actual hospital environment and no intervention, such as increasing or decreasing the number of data, increasing or decreasing the number of attributes, reduction, integration, imputation, transformation, or discretization, has been made on the incoming patient data. The original hospital data are classified for the diagnosis of anemia types and the accuracy of $84,97 \%$ achieved by using a deep learning algorithm.
\end{abstract}

Keywords: deep learning, anemia, classification

\section{Introduction}

The most common blood disease in Turkey and the world is the anemia group [1]-[4]. According to the World Health Organization, anemia is a condition in which the numbers of red blood cells and, consequently, oxygen-carrying capacities are inadequate to meet the body's physiological needs [2]. Anemia is also defined as a decrease in the concentration of erythrocyte mass or blood hemoglobin and hematocrit. Normal hemoglobin and hematocrit values vary according to age and sex. If hemoglobin and hematocrit values are below the lower limit of normal values for that age and sex, then there is anemia. In the study conducted by Kiassebaum et al. using data and resources from the World Health Organization's study on the global burden of disease 2010; 189 countries, 2 genders, and 20 different age groups were examined, and the global anemia frequency was calculated as $32.9 \%$. Most commonly, anemia is seen in children under the age of five and women. Iron deficiency anemia is the most common type of anemia [3]. Anemia affects the quality of life significantly. It is not only a disease but also a symptom that accompanies many diseases like cancer and kidney or liver failure. It can be very vital to treat it in many cases. To treat a patient, first, it is necessary to decide on the disease.

Various methods have been developed to assist experts in making decisions. When diagnosing anemia types, information about the patient such as age, gender, symptoms, chronic diseases, and the results of the complete blood count analysis are also important factors together. In many methods recommended for the diagnosis of anemia, only a few of the results of complete blood count analysis are used instead of using whole results. The reason for this is, it is not desirable to increase the number of attributes in many classification methods. Attributes are defining each instance of the data set in different aspects. Especially in medical problems, there can be a lot of attributes and lots of missing values. In the deep learning method, it is not a problem being the high size of attributes or instances. Whereas data analysis with deep learning is giving more successful results than the classical methods with both raw and largesized data. 
The method called deep learning is the name given to the deep neural network formed by adding many hidden layers to multilayer neural networks. Although it is not a new method, its use is becoming widespread and trendy nowadays. Deep learning algorithms have multi-input and multi-layered artificial neural network architecture. Deep neural networks' weight values are updated continuously for ensuring good results.

In this study, a multi-class probing solution was searched using deep learning architecture. It is aimed to develop a system that will enable the recognition of anemia in general practice conditions due to the increasing number of patients and the intention of the hospitals, as well as the difficulties in reaching the expert medical consultant. Running this system jointly with the tests required for diagnosing anemia in the primary health care services will help non-specialist personnel working in these health centers. At least based on this system, patients who need to be referred to the center can be selected faster and more accurately.

An overview of the problem of diagnosing anemia has been given in here, Introduction. The rest of the paper is organized as follows: Previous studies have been reviewed in Related Works. The anemia data used in this study has been described in Material and Method. Also, answers are given to questions like "what is anemia?" and "how to diagnose anemia?". Subsequently, methods used in the study are summarized and the architecture of the proposed deep learning system to diagnose anemia has been outlined. In Results, results of the models developed for this work are given and the evaluation has been made. Also, results are discussed. The motivation of this work and possible future works regarding this study has been given in Conclusion.

\section{Related Works}

Computer-aided decision-making and analysis is a widespread field in the medical area. A review of previous studies on the classification of anemia types was made, along with those conducted using similar methods but different data. One of the early studies about computer-aided anemia diagnosis is called PlanAlyzer. Beck et al., designed a computer-aided system for educational research in medical education. PlanAlyzer published in 1988 for diagnosis of heart disease [4] and anemia in 1989 [5] aimed to elucidate and criticize students' approach to diagnosing a widespread medical disorder. In a study published in 1993, Lyon et al. reported that this program was used for 7 years to teach the diagnosis of anemia and chest pain in cardiology and hematology departments of the Dartmouth School of Medicine after tests and assessments [6]. In 1960, Lipkin compared the data characteristics of hematological diseases and hospital data with the help of a digital computer. 49 patients and 20 diseases were selected, and hospital data were linked to the computer program. Differential diagnosis of hospital cases printed in written form [7]. In 1976, Engle and his colleagues introduced a computer program called HEME, which provided diagnostic analysis of hematological diseases to expert medical consultants. There are 40 hematological diseases in the system. The designed system was a rule-based system, and the Bayesian method was used [8]. Various algorithms have been developed to assist the doctor in the diagnosis of iron deficiency anemia, and their performances are successful [9] - [14]. Yavuz et al. conducted a study for the diagnosis of iron deficiency anemia in women. The data set used in the study includes six attributes including RBC, HGB, HCT, MCV, MCH, MCHC, and two classes and does not contain any missing values. Classification using ANNs and artificial immune system (AIS) was compared with that of KNN and the regression tree Gini algorithm. The performance of classification with the Gini-based decision tree method trained by the artificial immune system was more successful than the classification performance of the KNN method and ANNs [14]. Sanap et al. devised a classification that measured the severity of anemia using complete blood count reports and the C4.5 decision tree and support vector machine algorithms with the WEKA data mining tool. They included the ten numerical attributes of age, white blood cell count (WBC), hemoglobin (HGB), red blood cell count (RBC), hematocrit (HCT), mean cellular volume (MCV), mean cellular hemoglobin $(\mathrm{MCH})$, mean cellular hemoglobin concentration (MCHC), red cell distribution width (RDW) and platelet count (PLT) and four classes of anemia types: normocytic (anemia of chronic disease), microcytic (iron deficiency and thalassemia), macrocytic (Vitamin B12 and folate deficiency) and microcytic (renal anemia). The success rate of the C4.5 decision tree algorithm was $99.42 \%$, which surpassed the support vector machines with a success 
rate of 88.13\% [15]. In the study conducted by Amin and Habib, the full blood count parameters of WBC, RBC, HGB, HCT, MCV, MCHC, PLT, neutrophils (NEUT), lymphocytes (LYMP), monocytes (MONO), eosinophils (EO), and basophils (BO) and interpretation values of age were used as the data input. The classes included chronic anemia, eosinophilia, microcytic hypochromic anemia, normocytic anemia, neutrophil leukocytosis, neutrophil, unknown findings, and high erythrocyte sedimentation rate (ESR). They used the J48 decision tree, multi-layered perceptron, and naive Bayes as classifiers and achieved success rates of $97.16 \%, 86.55 \%$, and $70.28 \%$, respectively [16]. Iron deficiency anemia and thalassemia are two types of microcytic anemia that are at risk of confusion [17]. In a research article, a differential diagnosis of microcytic anemia was made with discriminant analysis using a training set consisting of 200 beta-thalassemia cases, 65 alpha-thalassemia cases, 170 iron deficiency anemia cases, and 45 cases having both iron deficiency anemia and beta-thalassemia [18]. Jamei and Talarposhti developed an artificial neural network (ANN) model with pattern-based input selection for iron deficiency anemia and $\beta$-thalassemia carriage discrimination. This method consisted of the decisionmaking ability of the ANNs combined with that of a human expert. Using complete blood count results, they devised a coefficient rule base and determined the multilayer perceptron neural network's input according to the calculated similarity. When compared with the performances of various authors using ANFIS, ANN, MLP, SVM, RBF, PNN, and KNN, their method was shown to have achieved the highest accuracy rate of 99.5\% [19]. In 2015, Kishore et al. published a study by determining the age, gender, HGB, MCV, MCH, HCT values as input, and iron deficiency and Vitamin B12 deficiency as output. They have developed a threaded ID3 approach by examining ID3 and non-threaded ID3 decision tree algorithms as methods. With the 480 data, they tested the system with both threaded and non-threaded ID3 and Gini algorithms and reported that the method they found was usable [20]. Shaik and Subashini presented a fuzzy logic approach for anemia diagnosis. They have use HGB, HCT, MCV, MCHC, WBC, Reticulocyte, Total Iron Binding Capacity (TIBC), Serum iron, and HSWC (hyper segmented white cells) laboratory test results as input parameters. As output, they use six anemia types which are Aplastic, Sideroblastic, Megaloblastic, Chronic, Myelophthisic, and Iron deficiency anemias [21]. Dalvi and Vernekar have made a study to determine the most suitable method to classify Red Blood Cells for anemia diagnosis. They use five ensemble learning methods which are AdaBoost, bagging, stacking, voting, and Bayesian boosting, and four classifiers which are the k-nearest neighbor, naïve Bayes, decision tree, and artificial neural network [22]. Belginova et al present a rule-based approach to the diagnosis of iron deficiency anemia. They have made a decision support system for expert medical consultants which includes patient data like identification, social status, medical history, complaints or sensations, medical indicators, and statistical information on the disease. By using these data an expert can decide the disease more accurately [23]. Dimauro et al. have made a study to predict the Hemoglobin value of patients with a non-invasive device that can analyze the image of the conjunctival region. They have tested the KNN classifier on 113 persons and obtained good results [24]. Using the complete blood count parameters Hasani and Hanani classified four different diseases which are Iron deficiency anemia, alpha-thalassemia trait, beta-thalassemia trait, and healthy. In this study, the combination of J48 and Naïve Bayes methods was found to be the highest success [25]. Guncar et al used machine learning methods in the diagnosis of hematological diseases. The data they use consists of laboratory blood tests of patients in the hematology clinic. SVM, Naive Bayes, and Random Forest were used as machine learning algorithms. They used ROC analysis for performance evaluation. They declared that the random forest algorithm gave the most effective result [26]. Ayyıldız and Tuncer used machine learning techniques consisting of SVM and KNN for the differential diagnosis of iron deficiency anemia and beta-thalassemia. They used 7 attributes which are RBC indices as input. They used the NCA algorithm for feature selection. They stated that they are $97 \%$ successful using ROC analysis as performance evaluation [27].

Deep learning methods also provide successful results in this respect. In a study published by Schmidhuber in 2015, deep learning methods, which have been popular in pattern recognition and machine learning in recent years, are examined and the history of shallow and deep architectures is explained [28]. Liu et al. explored deep learning architectures such as Auto Encoder, Convolutional Neural Network (CNN), Deep Belief Network, and Restricted Boltzmann Machines, and explained how they were used in speech recognition, pattern recognition, and computer vision areas, in their work [29]. Deep learning methods are often used in the analysis of medical images. In a study by Litjens et al 
performed in 2017, studies in medical fields such as brain, eye, chest, skeleton, and pathology that use images in important tasks such as image classification, object recognition, and segmentation are surveyed [30]. Miotto et al proposed a method of non-consultant deep feature learning, modeling clinical prediction using electronic health records. They called their work the deep patient, and it contains raw data such as clinical notes, diagnosis, medications, and laboratory tests. Using this data, all the diseases that each patient has had over time are labeled. Thus, by reviewing the patients' past records, both diseases have been labeled and possible future diseases can be estimated [31]. Yu et al stated that they obtained higher accuracy in a shorter time by using a deep learning algorithm for white blood cell classification [32]. Xu et al made a study to diagnose sickle cell disease. They have developed a system to examine RBC (Red Blood Cell) images and used CNN (Convolutional Neural Networks) to classify RBC [33]. Kimura et al developed a deep-learning-based image analysis system to make a differential diagnosis of aplastic anemia and MDS. They divided the data set into 17 cell types and 97 abnormal morphological features. Then they created a CNN module to classify them. They used the ROC curve for performance measurement and the AUC (area under the curve) was found as 0.99 [34]. Alsheref and Gomaa conducted a study on blood disease prediction with machine learning methods. They developed a new data set consisting of 668 patients' blood tests, containing 28 parameters and 4 classes. They used the WEKA tool for applying machine learning algorithms and found the highest result of $98.16 \%$ with the logit boost method [35]. Çil, Ayyıldız, and Tuncer have developed a decision support system to make a differential diagnosis of iron deficiency anemia and beta-thalassemia. They performed a $95.59 \%$ successful classification using logistic regression, KNN, SVM, ELM, and RELM algorithms. They used HGB, RBC, HCT, MCV, MCH, MCHC, and RDW parameters of 342 patients [36]. Varghese conducted a study highlighting the importance of machine learning methods in the classification of blood cells and blood diseases. Varghese analyzed lots of articles and claims that CNN and SVM methods give high success results [37]. Alzubaidi et al conducted a study to diagnose sickle cell anemia by classifying RBC shapes. They recognized RBCs as 3 classes as normal, sickle cell, and other blood content. In their study, they proposed 3 different deep learning models. They used transfer learning and data augmentation since there was no training data. They stated that their models made high accuracy classification [38]. Devunooru et al. conducted a study for use in the diagnosis of brain tumors. They performed the segmentation process of my brain MRI images using deep learning methods and obtained successful results [39]. Göceri has conducted a study that will automate the classification of dermatological diseases. Within the scope of the study, he finds and identifies the lesioned areas on the skin from color digital photographs and classifies them [40]. Kıliçarslan et al. developed a deep learning-based hybrid method for the classification of nutritional anemia diseases. At the end of the classification process, the data were divided into 3 classes: HGB-anemia, nutritional anemia, and patients without anemia. They enriched the stacked autoencoder and CNN methods, deep learning algorithms, genetic algorithms, and successful results [41]. Yeruva et al., in their study, determined the type of deterioration of hemoglobin structure from the shapes of red blood cells using deep neural network methods. They classified it according to the shape of the cells as sickle cell, thalassemia, and normal. As a method, they compared the multilayer perceptron method with other machine learning methods and stated that they were successful [42]. Gupta et al. conducted a study that detected the presence of covid 19 pneumonia, a new disease that affects millions of people around the world. They determined whether the patient had covid 19 from chest x-ray images with the deep convolution network method and said that they were successful [43]. Venugopalan et al. conducted a study to determine the stage of Alzheimer's disease. They emphasized the success of deep learning methods by comparing deep learning methods with other machine learning methods in identifying various regions of the brain on brain MRI images [44]. Atila et al. conducted a study that used deep learning architecture to classify diseases observed in the leaves of plants [45].

When these studies are examined, it is seen that studies are carried out to diagnose one or more general types of anemia like microcytic, normocytic, and macrocytic anemias in [15], [16] or thalassemia and iron deficiency anemia in [17], [18] and [19]. Although various research were carried out up to date, it has not been encountered that diagnosing 14 different anemia types related to study in the literature. Thus, in this study, a multi-class probing solution for diagnosing 14 different types of anemia which are described in WHO's International Disease Classification (ICD) Codes were investigated by using deep learning architecture. Besides, in most of the works above, the attributes used are mostly limited to a 
few blood parameters only. In this study, 30 different attributes that are used by the expert medical consultant to diagnose the diseases have been used. Also, the data used in this study are completely original and include age, gender, chronic diseases, and symptoms as well as blood parameters.

\section{Material and Method}

The present study, it is aimed to develop a system that will enable the recognition of anemia in general practice conditions due to the increasing number of patients and the intention of the hospitals, as well as the difficulties in reaching the expert medical consultant. For this purpose, real patient data and the advice of an expert medical consultant are needed. For the diagnosis of anemia, information like which feature is needed, which method should be followed to diagnose which disease has been determined with the expert medical consultant, and the necessary data required could only be obtained in this way from the ethics committee. Once ethical approval is obtained, data were transferred from a clinical database to the program interface shown in Figure 1.

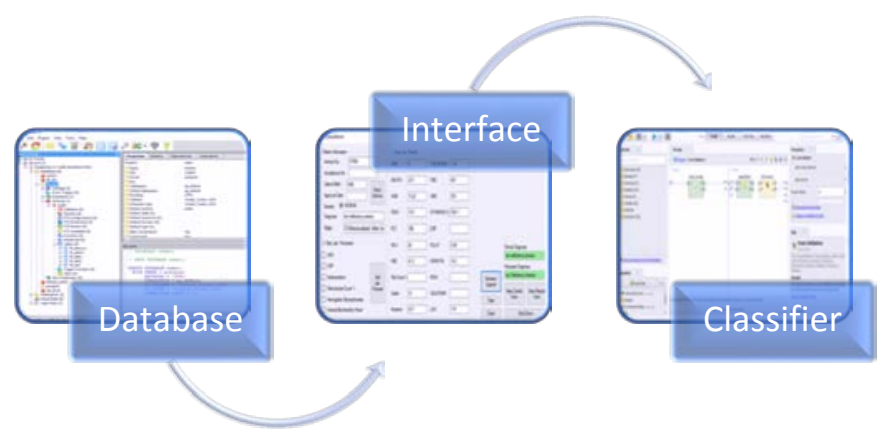

Figure 1. The Design of the Proposed System.

As seen in Figure 1 real patient data was transferred from a database to the program interface. With the help of the interface, data are given to the deep learning environment to classify anemia types. The transactions made during this process do not involve any interference with the data. Only data stored in multiple tables in the database are combined into a single table. After the data were obtained and prepared with the help of the program interface, a deep learning model was developed for the classification process. To demonstrate, the diagram of the present study is shown in Figure 2.

In this step, the training and the testing process were conducted for the deep learning method. Finally, the developed model was recorded and tested on new data and the performance was evaluated by accuracy, classification error, kappa, weighted mean recall and precision, absolute and relative errors, root means squared error (RMSE) and Receiver Operating Characteristic (ROC) analysis method. The flowchart diagram of the entire study is shown in Figure 2.

\subsection{Dataset Definition}

The data used in the present study are actual patient data obtained from the Duzce University Research and Practice Hospital with permission from the ethics committee. To obtain the approval of the ethics committee, every detail had to be determined. Therefore, all the attributes of the data were to be announced to the committee. So, it has not been needed for any pre-processing other than digitizing data and no intervention was required. Since there was no process for changing the content and quality of the data, in the present study a completely original dataset was used in which not even any numerical intervention was made. The data obtained by the approval of the ethics committee includes 34 attributes. Three of which are located in the raw dataset and which enable us to organize our data are not used as attributes. These are archive number, accept number, and approval date. Archive number is a unique attribute to define the patient in the hospital records. By the accept number and the approval date of the patient, it can be reached that how often the same patient applies to the clinic. These data are not included in the dataset. All the attributes used in the dataset are shown in detail in Table 1. 


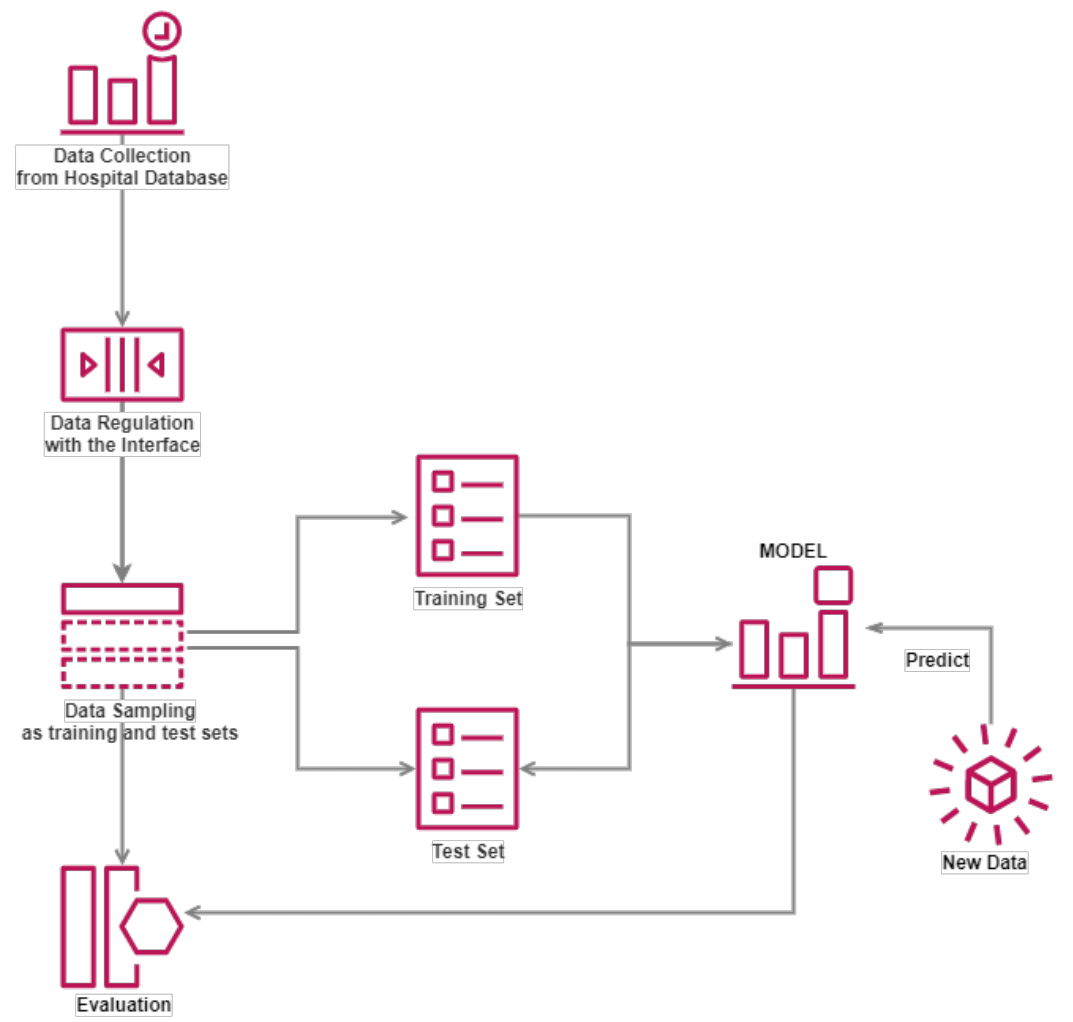

Figure 2. The Flow Chart of the Whole Study.

Information such as age, gender, presence of symptoms, and chronic diseases of the patients are attributes that play an important role in determining the type of anemia. Other Information contains the medical history of the patient. Bilirubin values in blood analysis are used to assess liver and gall bladder functions. C Reactive Protein (CRP) provides information about the presence of inflammation in the body. Iron value in the blood is used in the evaluation of all kinds of anemia, iron deficiency, and iron poisoning. Ferritin value is used in the diagnosis of iron deficiency anemia, chronic disease anemia, and thalassemia. It is also important for following iron loading treatment. Folate means folic acid value in the blood and is used in the evaluation of megaloblastic and macroscopic anemia. Also, it is used to observe the treatment of folate deficiency anemia. Hematocrit (HCT) value shows the amount of hemoglobin and erythrocytes existence in the blood. Hemoglobin (HGB) value shows the total amount of hemoglobin existence in blood. It is the first value to investigate in complete blood count parameters that indicate anemia. Creatinine value in the blood is used in the evaluation of kidney functions. Lactate dehydrogenase (LDH) is an enzyme that helps the body produce energy and is released into the bloodstream in situations such as sickness and injury in that cells are damaged. Thus, the value of the LDH in the blood increases. It is an important parameter for the detection of hemolytic anemia from anemia types. Mean Cell Hemoglobin (MCH) shows the total amount of hemoglobin in erythrocytes. Mean Cell Hemoglobin Concentration (MCHC) is the percentage of hemoglobin concentration in erythrocytes. Mean Corpuscular Volume (MCV) is the average size of red blood cells carrying oxygen. NEUT\# value is the number of neutrophils in the blood. NEUT\% value is the percentage of neutrophils in the blood. Procalcitonin (PCT) is a hemogram parameter that indicates the percentage of thrombocytes in the blood. Platelet Dispersion Width (PDW) is another hemogram parameter that shows the distribution range of the sizes of thrombocytes. The lower value of PDW means that the sizes of the platelets are very similar, and the higher value of PDW means that the sizes of the platelets are very different in size from each other. PLT value is the number of platelets that allow blood to clot. Red Blood Cell (RBC) is the amount of erythrocytes presence in red blood cells. Red cell Distribution Width (RDW) shows the distribution width of erythrocytes in the blood. Total iron-binding capacity (TIBC) and unbound iron-binding capacity (UIBC) are also important parameters to diagnose anemia types. Vitamin B12 is an essential vitamin for hematopoiesis and normal neuronal functions. In case of a lack of Vitamin B12, Vitamin B12 deficiency anemia may be considered. The White blood cell (WBC) value 
is the number of leucocytes in the white blood cells which is responsible for the body's defense and immunity system [46]-[50].

Table 1 List of the Attributes in the Dataset

\begin{tabular}{|c|c|c|c|c|}
\hline Attribute Name & Type & Min. & Max. & Avg. \\
\hline Age & Numeric & 4 & 91 & 54,54 \\
\hline Gender & Polynomial & - & - & - \\
\hline Chronic Disease & Polynomial & - & - & - \\
\hline Symptoms & Polynomial & - & - & - \\
\hline Other Information & Polynomial & - & - & - \\
\hline CRP (C Reactive Protein) & Numeric & 0 & 27,60 & 1,24 \\
\hline D. Bilirubin (Direct Bilirubin) & Numeric & 0 & 2,45 & 0,22 \\
\hline Iron & Numeric & 4 & 377 & 79 \\
\hline Ferritin & Numeric & 0 & 899,40 & 111,76 \\
\hline Folate & Numeric & 2,61 & 25,20 & 10,64 \\
\hline HCT (Hematocrit) & Numeric & 11 & 64,50 & 35,48 \\
\hline HGB (Hemoglobin) & Numeric & 3,80 & 22,90 & 11,79 \\
\hline I. Bilirubin (Indirect bilirubin) & Numeric & $-0,13$ & 5,01 & 0,49 \\
\hline Creatinine & Numeric & 0,23 & 8 & 0,92 \\
\hline LDH (Lactate Dehydrogenase) & Numeric & 57 & 3685 & 227,63 \\
\hline MCH (Mean Cell Hemoglobin) & Numeric & 13,99 & 45,20 & 27,54 \\
\hline MCHC (Mean Cell Hemoglobin Concentration) & Numeric & 25,60 & 38,20 & 33,13 \\
\hline MCV (Mean corpuscular volume) & Numeric & 49 & 126,60 & 82,87 \\
\hline NEUT\# (Neutrophils) & Numeric & 0 & 47,83 & 4,12 \\
\hline NEUT\% & Numeric & 0 & 95,20 & 57,77 \\
\hline PCT & Numeric & 0 & 1015 & 1,10 \\
\hline PDW & Numeric & 8,60 & 31,50 & 14,82 \\
\hline PLT (Platelets) & Numeric & 2 & 1239 & 259,12 \\
\hline RBC (Red Blood Cell) & Numeric & 1,24 & 8,84 & 4,33 \\
\hline RDW (Red cell Distribution Width) & Numeric & 11,20 & 38,20 & 17,21 \\
\hline T. Bilirubin (Total Bilirubin) & Numeric & $-0,02$ & 5,73 & 0,72 \\
\hline TIBC (Total iron-binding capacity) & Numeric & 104 & 697 & 354,14 \\
\hline UIBC (Unbound iron-binding capacity) & Numeric & -9 & 676 & 275,14 \\
\hline Vitamin B-12 & Numeric & 30 & 1941 & 495,33 \\
\hline WBC (White blood cell) & Numeric & 0,70 & 431,33 & 8,07 \\
\hline Diagnosis & Polynomial & - & - & - \\
\hline
\end{tabular}

There are 1477 data in the data set. The distribution of these data according to diagnosis is given in Table 2. As it is seen, in Duzce which is a province in Turkey's western Black Sea region, the distribution

Table 2 Distribution of the Diagnoses in the Dataset

\begin{tabular}{|c|c|c|c|}
\hline ICD 10 Code & Diagnosis & Count & Percentage \\
\hline D50 & Iron deficiency anemia & 468 & $31,69 \%$ \\
\hline D64 & Anemia & 283 & $19,16 \%$ \\
\hline D63 & Anemia of chronic disease & 241 & $16,32 \%$ \\
\hline--- & Other & 132 & $8,94 \%$ \\
\hline D46 & Myelodysplastic Syndrome & 127 & $8,60 \%$ \\
\hline D56 & Thalassemia & 68 & $4,60 \%$ \\
\hline D51 & Vitamin B12 deficiency anemia & 38 & $2,57 \%$ \\
\hline D59 & Hemolytic anemia & 35 & $2,37 \%$ \\
\hline D50-D51 & Iron and vitamin B12 deficiency anemia & 29 & $1,96 \%$ \\
\hline D50-D51-D52 & Iron, folate and vitamin B12 deficiency anemia & 16 & $1,08 \%$ \\
\hline D50-D52 & Iron and folate deficiency anemia & 13 & $0,88 \%$ \\
\hline D51-D52 & Folate and vitamin B12 deficiency anemia & 12 & $0,81 \%$ \\
\hline D52 & Folate deficiency anemia & 11 & $0,74 \%$ \\
\hline D57 & Carriage of thalassemia & 4 & $0,27 \%$ \\
\hline & Total & 1477 & $100 \%$ \\
\hline
\end{tabular}


of diseases associated with anemia is not regular and balanced. There are 977 female and 500 male patients in the data set. Women are known to have a high prevalence of anemia. These data also confirm the nature of this situation. Iron deficiency anemia, which constitutes $31,69 \%$ of the dataset, is the most common type of anemia seen in the Duzce region. The least common disease is the thalassemia trait. However, it should be kept in mind that to obtain these data for use in our study, ICD codes are limited between D50 and D64.9, and attributes are limited to 34 different features. Therefore, the attributes needed to diagnose anemia-associated diseases were selected by the expert medical consultant's recommendation.

The anemia types listed here are the 14 most common types of anemia in the region of Duzce in Turkey where data are taken. As the data are taken from the hospital, all the patients in the dataset have at least one hematologic disorder. The "other" patient group does not consist of healthy individuals. For this reason, it is not considered a control group. Since they are suffering from other hematologic disorders outside the anemia group, the use of the "other" term was considered appropriate. In the last case, classification with 31 attributes including the diagnosis column shown in Table 2 was performed.

\subsection{Deep Learning Method}

Artificial neural networks are one of the most widely used classification methods in the literature. It is an artificial learning model designed by inspiring the nervous system in living things. The inspired nervous system consists of neurons and synaptic connections of neurons. When the mathematical model of a neuron is designed, the synaptic connections are represented by connection weights. Each neuron should have input connections, bias values, activity level, output connections, and an output value. Equation (1) shows the output connection of a neuron where $\boldsymbol{a}_{\boldsymbol{i}}$ indicates the input value, $\boldsymbol{w}_{\boldsymbol{i}}$ indicates the weight value of each input, and $b$ indicates the bias value for the neuron.

$$
f(x)=f\left(\sum_{i=1} w_{i} a_{i}+b\right.
$$

The output is created by making calculations in the equation for each input value to a neuron. The neuron value is reflected in the transfer function $\mathrm{f}(\mathrm{x})$ as the sum of the input signals multiplied by the weights. This output is passed through the activation function and transmitted to the next layer. The learning capacity of an artificial neuron is determined by arranging the weights of the chosen learning algorithm $[51,52]$.

The artificial neural network usually consists of an input layer, a hidden layer, and an exit layer. In deep networks, both the number of these layers and the number of neurons in the layers should be higher. The deep network used in this study is based on a multi-layer feed-forward neural network trained by stochastic gradient descent using a backpropagation algorithm. The proposed model includes an input layer containing 30 neurons, 5 hidden layers each containing 50 neurons, and an output layer containing 14 neurons. The network structure of the proposed model is shown in Figure 3.

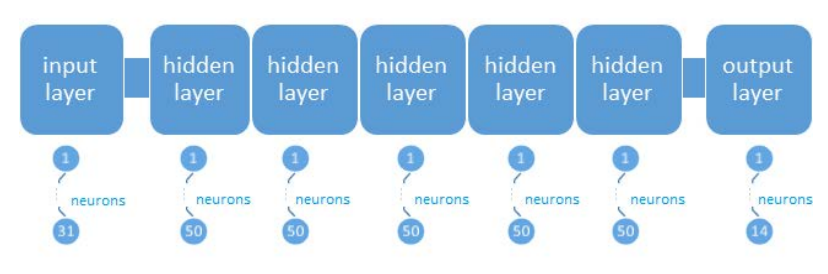

Figure 3. The Basic Structure of the Proposed Model.

The number of hidden layers can be very numerous. The activation functions and numbers of the neurons in the hidden layer are determined by the structure of the problem [53]. For this work, hyperbolic tangent function (tanh), rectifier linear unit, maxout, and exponential rectifier linear unit functions are selected as the activation functions. The hyperbolic tangent function is a version of scaled and shifted sigmoid function. Equation (2) shows the sigmoid function where $\mathrm{x}$ indicates inputs. 


$$
\delta(x)=\frac{1}{1-e^{-x}}
$$

The rectifier linear unit function selects the maximum values of $(0, x)$ where $x$ is the input value. The maxout function selects the maximum coordinate of the input vector. The exponential rectifier linear unit function is the exponential version of the rectifier linear unit function.

The network structure of the proposed method is seen in Figure 4. In the present study, 5 hidden layers are used, each of the hidden layers consists of 50 neurons inside.

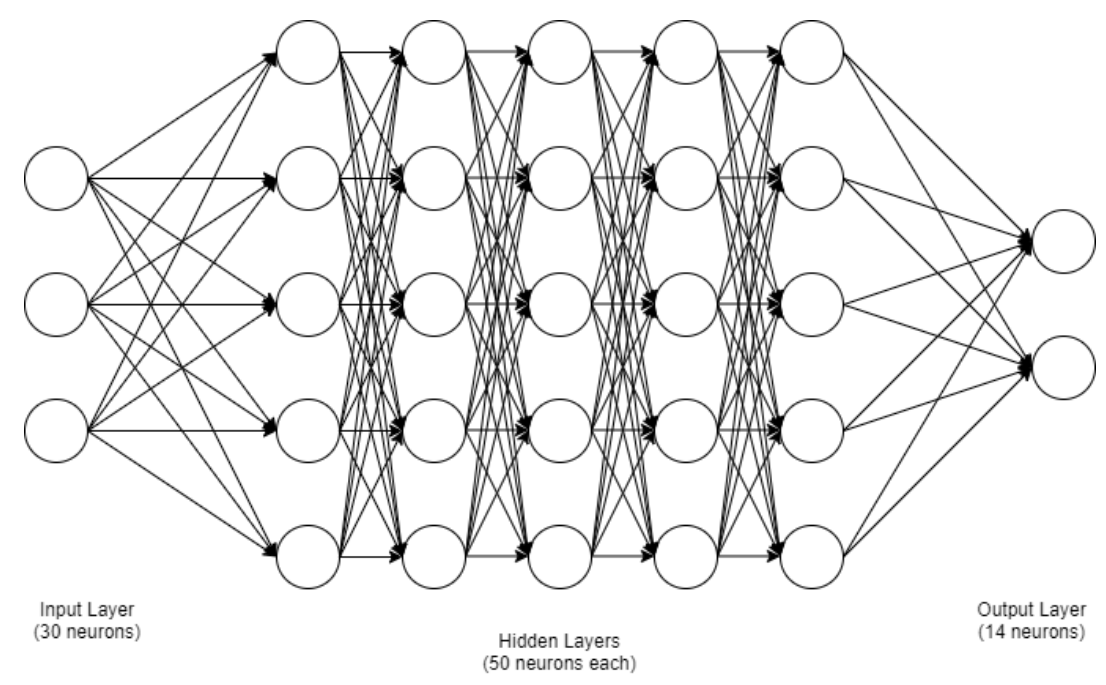

Figure 4. The Network Structure of the Proposed Method.

Deep learning is a popular method in recent years because it gives successful results on big and raw data without pre-processing. With the development of technology, there is a lot of data both in terms of size and quality and it takes time to perform pre-processing or cleaning on these data. Classical methods often cause memory errors due to the size of the data and are not sufficient. In such situations, deep learning methods are preferred, which process data instantly and produce results, and thus use less memory. While deep learning is widely used in the areas of object recognition and image processing which are using big sizes of memory it is becoming increasingly common to use with numerical or polynomial data in the important scientific fields that affect human health, such as medicine. This study contributes to the advancement of the use of deep learning in medical areas.

\subsection{Evaluation}

For the present study, k-fold cross-validation method was used to evaluate the proposed method. In the $\mathrm{k}$-fold cross-validation method, the dataset was divided into $\mathrm{k}$ different subsets. When a group is a test set, the remaining groups are used as training sets. In this way, all the combinations were tested so that each of the $\mathrm{k}$ data subsets is used as once a test set. As a result, a performance value was found by taking the average of each result.

Receiver operating characteristic (ROC) analysis is used for performance measurement in this study. The ROC analysis is an effective method for measuring the performance of machine learning and data mining techniques [54]. ROC curve is used to evaluate the equilibrium between accuracy, sensitivity and specificity. Accuracy, sensitivity and specificity values are basically calculated as the formulas given in Equations (3), (4) and (5) respectively.

$$
\text { Accuracy }=\frac{T P+T N}{(T P+T N+F P+F N)}
$$




$$
\begin{aligned}
& \text { Sensitivity }=\frac{T P}{(T P+F N)} \\
& \text { Specificity }=\frac{T N}{(T N+F P)}
\end{aligned}
$$

Expressions which are used in the formulas (3), (4), and (5) are given below:

TP (True Positive): Number of the instances where the predicted value is positive, and the real value is positive.

TN (True Negative): Number of the instances where the predicted value is negative, and the real value is negative.

FP (False Positive): Number of the instances where the predicted value is positive, and the real value is negative.

FN (False Negative): Number of the instances where the predicted value is negative, and the actual value is positive.

The ROC curve of the presented method used in the present study is shown in the Figure 5.

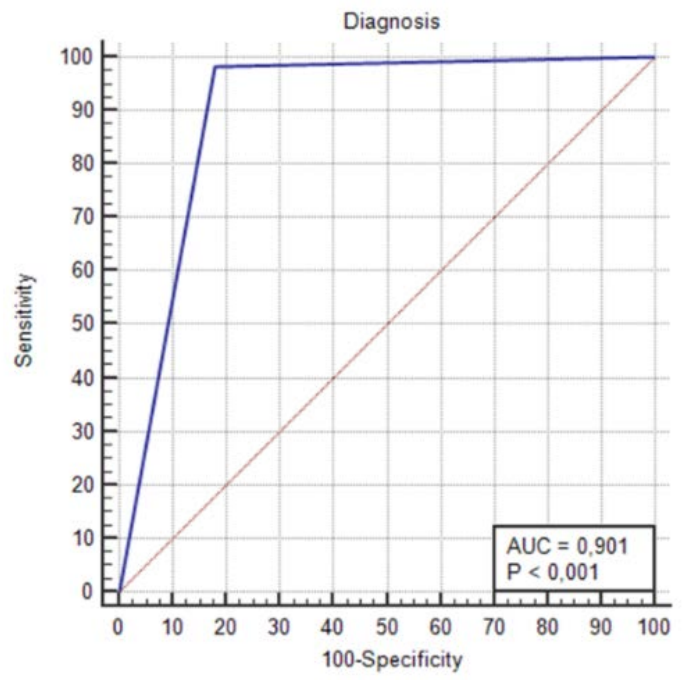

Figure 5. The ROC Curve of the Presented Method.

The area under the ROC curve is an important parameter to measure the success of classification methods. The closer this value, which defines as AUC -Area under the curve, to 1, the more accurate the prediction has been made. For the success of the classification to be acceptable, it is desired to form a curve in the upper left part of the red line in the graph shown in Figure 5. Otherwise, the classification is considered unsuccessful. If the AUC value is 1, all classes are correctly estimated. If the AUC value is 0 , it means that no class is correctly predicted [54].

The model also evaluated by accuracy, classification error, kappa, weighted mean recall and precision, absolute and relative errors, root means squared error methods. Accuracy is the percentage of correctly predicted data. Classification error is te percentage of the data that predicted incorrectly. Kappa statistics is calculated as the Equation (6):

$$
\mathrm{K}=\frac{P(a)-P(r)}{1-P(r)}
$$

Here, $\boldsymbol{P}(\boldsymbol{a})$ represents correctly predicted data, while $\boldsymbol{P}(\boldsymbol{r})$ represents correctly predicted data by chance. If $\mathrm{K}$ is 1 , it means that the data was predicted completely correctly, while $\mathrm{k}$ is 0 means that the data was predicted correctly by pure chance. The precision is the rate at which the predicted positive class is actually positive. The recall is the ratio of correctly predicted true positives [55]. The absolute and relative errors and root mean squared error (RMSE) are also significant methods used in performance 
measurements. In the present study, performance evaluation is made with the methods mentioned above and the results are given in the next section with Tables 6-8.

\section{Results and Discussion}

In the presented study, a system that can diagnose anemia like an expert medical consultant is designed. For this purpose, the deep learning method is tested with the original dataset including real patient data. The data used in the present study are original patient data obtained from the Research and Practice Hospital of Duzce University with permission taken from the ethics committee of the hospital. The attributes used are shown in Table 1. The information about how often the same patient applies to the clinic is reached by using the date of approval, the archive, and the acceptance numbers of patients, which are not included as attributes. Also, information such as age, gender, symptoms, and other chronic diseases of the patients are attributes that play an important role in determining the type of anemia. Other attributes in Table 1 are obtained from laboratory test results of complete blood count. Diagnosis decision is made by the expert medical consultant. To determine the presence of anemia, firstly the hemoglobin (HGB) value is examined. If HGB is less than $13 \mathrm{~g} / \mathrm{dL}$ for men and $12 \mathrm{~g} / \mathrm{dL}$ for women, then there is anemia. In the next step, the Mean Corpuscular Volume (MCV) value is examined. Anemia can be macrocytic if MCV is greater than 100, normocytic if MCV is between 80 and 100, or microcytic if MCV is less than 80. After identifying the first phase the expert may require further investigation and advanced tests for a definite diagnosis. For example, if the iron value is low and creatinine value is normal, iron deficiency anemia can be considered as the diagnosis. If creatinine value is also low, then anemia of chronic disease can be considered as the diagnosis. Detailed types or causes of anemia are shown in Figure 6.

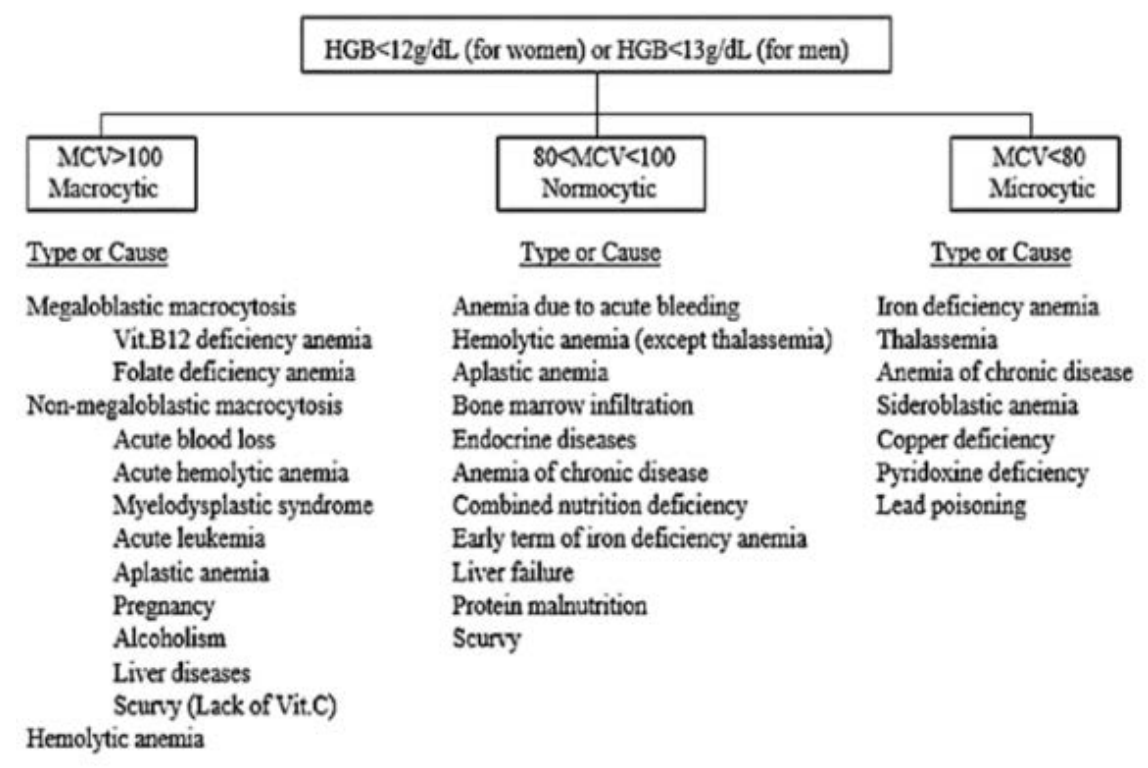

Figure 6. The Classification of Anemia According to the Erythrocyte Morphology [50].

The present study aimed to diagnose 14 different types of hematological disorders, most of which are anemia groups. The diseases and ICD-10 codes are seen in Table 2 . The total numbers of attributes are 31 , the total numbers of instances in the data set are 1477 and the total classes are 14 . When the data is classified by using the deep learning algorithm, the results shown in Table 3 are obtained.

As seen in Table 3, the highest accuracy and minimum classification error achieved by using 10-fold cross-validation with the deep learning algorithm. In the cross-fold validation method, the data set is divided into $\mathrm{k}$ different subsets. When a subset is a test set, the remaining $\mathrm{k}-1$ subsets are set as a training set. In this way, all the combinations are tested so that each of the $\mathrm{k}$ subsets is once a test set, and as a result, a performance value is found by taking the average of each result. Also, the ROC analysis method 
was used for performance measurement. The ROC curve of the proposed method is seen in Figure 5. Table 4 and Table 5 show the confusion matrixes of the proposed method.

Table 3. Performance of the proposed method.

\begin{tabular}{|c|c|c|}
\hline Deep Learning & Accuracy & Error \\
\hline Split validation (0,7) & $81,00 \%$ & $19,00 \%$ \\
\hline Cross-validation (5-fold) & $83,07 \%$ & $16,93 \%$ \\
\hline Cross-validation (7-fold) & $83,28 \%$ & $16,72 \%$ \\
\hline Cross-validation (10-fold) & $84,97 \%$ & $15,03 \%$ \\
\hline Cross-validation (12-fold) & $84,29 \%$ & $15,71 \%$ \\
\hline Cross-validation (15-fold) & $84,50 \%$ & $15,50 \%$ \\
\hline
\end{tabular}

Table 4. Confusion matrix of 14 classes (Split Validation).

\begin{tabular}{|c|c|c|c|c|c|c|c|c|c|c|c|c|c|c|c|}
\hline & \multicolumn{15}{|c|}{ True } \\
\hline Pred. & Ane. & IDA & CoT & $\begin{array}{c}\text { F-B12- } \\
\text { DA }\end{array}$ & ACD & B12-DA & Other & I-F-DA & MDS & $\begin{array}{c}\text { I-B12- } \\
\text { DA }\end{array}$ & HA & $\begin{array}{c}\text { I-F-B12- } \\
\text { DA }\end{array}$ & FDA & Thal. & $\begin{array}{l}\text { class } \\
\text { prec. }\end{array}$ \\
\hline Ane. & 60 & 13 & 0 & 1 & 7 & 3 & 1 & 1 & 0 & 0 & 2 & 0 & 1 & 1 & 66.6 \\
\hline IDA & 14 & 121 & 1 & 0 & 3 & 0 & 1 & 1 & 0 & 2 & 0 & 1 & 1 & 3 & 81.7 \\
\hline CoT & 0 & 0 & 0 & 0 & 0 & 0 & 0 & 0 & 0 & 0 & 0 & 0 & 0 & 0 & 0.0 \\
\hline F-B12-DA & 0 & 0 & 0 & 3 & 0 & 0 & 0 & 0 & 0 & 0 & 0 & 0 & 0 & 0 & 100 \\
\hline ACD & 6 & 2 & 0 & 0 & 58 & 0 & 1 & 0 & 0 & 0 & 1 & 0 & 0 & 1 & 84.0 \\
\hline B12-DA & 1 & 2 & 0 & 0 & 0 & 7 & 0 & 0 & 0 & 0 & 2 & 0 & 0 & 0 & 58.3 \\
\hline Other & 3 & 0 & 0 & 0 & 4 & 1 & 37 & 0 & 0 & 0 & 0 & 0 & 0 & 0 & 82.2 \\
\hline I-F-DA & 0 & 0 & 0 & 0 & 0 & 0 & 0 & 2 & 0 & 0 & 0 & 0 & 0 & 0 & 100 \\
\hline MDS & 0 & 0 & 0 & 0 & 0 & 0 & 0 & 0 & 38 & 0 & 0 & 0 & 0 & 0 & 100 \\
\hline I-B12-DA & 0 & 0 & 0 & 0 & 0 & 0 & 0 & 0 & 0 & 7 & 0 & 0 & 0 & 0 & 100 \\
\hline HA & 0 & 0 & 0 & 0 & 0 & 0 & 0 & 0 & 0 & 0 & 5 & 0 & 0 & 0 & 100 \\
\hline I-F-B12-DA & 0 & 0 & 0 & 0 & 0 & 0 & 0 & 0 & 0 & 0 & 0 & 4 & 0 & 0 & 100 \\
\hline FDA & 1 & 0 & 0 & 0 & 0 & 0 & 0 & 0 & 0 & 0 & 0 & 0 & 1 & 0 & 50.0 \\
\hline Thal. & 0 & 2 & 0 & 0 & 0 & 0 & 0 & 0 & 0 & 0 & 0 & 0 & 0 & 15 & 88.2 \\
\hline class recall & 70.5 & 86.4 & 0.0 & 75.0 & 80.5 & 63.6 & 92.5 & 50.0 & 100 & 77.7 & 50.0 & 80.0 & 33.3 & 75.0 & \\
\hline
\end{tabular}

Abbreviations: IDA - Iron deficiency anemia, Ane. - Anemia, ACD - Anemia of chronic disease, Other - Other, MDS - Myelodysplastic Syndrome, Thal. - Thalassemia, B12DA - Vitamin B12 deficiency anemia, HA - Hemolytic anemia, I-B12-DA - Iron and vitamin B12 deficiency anemia, I-F-B12-DA - Iron, folate, and vitamin B12 deficiency anemia, I-F-DA - Iron and folate deficiency anemia, F-B12-DA Folate and vitamin B12 deficiency anemia, FDA - Folate deficiency anemia, CoT - Carriage of thalassemia.

As seen in Table 4, MDS is the best-predicted class with a 100\% accuracy rate, and CoT is found as the worst predicted class with a $0 \%$ accuracy rate. The average accuracy rate is $81 \%$ for split validation. And as seen in Table 5, again MDS is the best-predicted class with a 98,43\% accuracy rate and FDA is found as the worst predicted class with a $0 \%$ accuracy rate. The average accuracy rate for 10 -fold crossvalidation is $84,97 \%$.

Table 5. Confusion matrix of 14 classes (10-fold cross-validation).

\begin{tabular}{|c|c|c|c|c|c|c|c|c|c|c|c|c|c|c|c|}
\hline \multirow[b]{2}{*}{ Pred. } & \multicolumn{15}{|c|}{ True } \\
\hline & Ane. & IDA & CoT & $\begin{array}{c}\text { F-B12- } \\
\text { DA } \\
\end{array}$ & ACD & B12-DA & Other & I-F-DA & MDS & $\begin{array}{c}\text { I-B12- } \\
\text { DA }\end{array}$ & HA & $\begin{array}{c}\text { I-F-B12- } \\
\text { DA }\end{array}$ & FDA & Thal. & $\begin{array}{l}\text { class } \\
\text { prec. }\end{array}$ \\
\hline Ane. & 215 & 28 & 0 & 2 & 20 & 5 & 12 & 2 & 1 & 3 & 2 & 1 & 3 & 3 & 72.3 \\
\hline IDA & 42 & 430 & 1 & 1 & 12 & 8 & 3 & 2 & 1 & 9 & 1 & 1 & 2 & 3 & 83.3 \\
\hline CoT & 0 & 0 & 3 & 0 & 0 & 0 & 0 & 0 & 0 & 0 & 0 & 0 & 0 & 0 & 100 \\
\hline F-B12-DA & 0 & 0 & 0 & 7 & 0 & 0 & 0 & 0 & 0 & 0 & 0 & 0 & 0 & 0 & 100 \\
\hline ACD & 17 & 4 & 0 & 1 & 206 & 1 & 4 & 0 & 0 & 0 & 1 & 0 & 0 & 0 & 88.0 \\
\hline B12-DA & 1 & 2 & 0 & 0 & 0 & 22 & 0 & 0 & 0 & 1 & 0 & 0 & 0 & 0 & 84.6 \\
\hline Other & 6 & 2 & 0 & 1 & 0 & 2 & 112 & 0 & 0 & 0 & 1 & 0 & 1 & 0 & 89.6 \\
\hline I-F-DA & 0 & 0 & 0 & 0 & 0 & 0 & 0 & 9 & 0 & 0 & 0 & 0 & 0 & 0 & 100 \\
\hline MDS & 0 & 2 & 0 & 0 & 2 & 0 & 1 & 0 & 125 & 0 & 0 & 0 & 1 & 0 & 95.4 \\
\hline I-B12-DA & 0 & 0 & 0 & 0 & 0 & 0 & 0 & 0 & 0 & 16 & 0 & 0 & 0 & 0 & 100 \\
\hline HA & 0 & 0 & 0 & 0 & 0 & 0 & 0 & 0 & 0 & 0 & 30 & 0 & 0 & 0 & 100 \\
\hline I-F-B12-DA & 0 & 0 & 0 & 0 & 0 & 0 & 0 & 0 & 0 & 0 & 0 & 14 & 0 & 0 & 100 \\
\hline FDA & 1 & 0 & 0 & 0 & 0 & 0 & 0 & 0 & 0 & 0 & 0 & 0 & 4 & 0 & 80.0 \\
\hline Thal. & 1 & 0 & 0 & 0 & 1 & 0 & 0 & 0 & 0 & 0 & 0 & 0 & 0 & 62 & 96.8 \\
\hline class recall & 75.9 & 91.8 & 75.0 & 58.3 & 85.4 & 57.8 & 84.8 & 69.2 & 98.4 & 55.1 & 85.7 & 87.5 & 36.3 & 91.1 & \\
\hline
\end{tabular}

Table 6 shows the success rates obtained by using the Tanh, Rectifier, Maxout, and ExpRectifier activation functions after 50 epochs. 
Table 6. Success rates of the method after 50 epochs.

\begin{tabular}{|c|c|c|c|c|}
\hline 50 epochs & Tanh & Rectifier & Maxout & ExpRectifier \\
\hline Accuracy (\%) & 81,38 & 78,61 & 75,70 & 81,04 \\
\hline Classification error (\%) & 18,62 & 21,39 & 24,30 & 18,96 \\
\hline Kappa & 0,768 & 0,734 & 0,697 & 0,765 \\
\hline Recall (\%) & 66,48 & 58,74 & 54,82 & 65,89 \\
\hline Precision (\%) & 71,90 & 62,96 & 60,45 & 70,69 \\
\hline Absolute error & 0,195 & 0,221 & 0,254 & 0,195 \\
\hline Relative error (\%) & 19,54 & 22,07 & 25,38 & 19,46 \\
\hline RMSE & 0,413 & 0,446 & 0,479 & 0,413 \\
\hline
\end{tabular}

Table 7 shows the success rates obtained by using the Tanh, Rectifier, Maxout, and ExpRectifier activation functions after 100 epochs.

Table 7. Success rates of the method after 100 epochs.

\begin{tabular}{|c|c|c|c|c|}
\hline 100 epochs & Tanh & Rectifier & Maxout & ExpRectifier \\
\hline Accuracy (\%) & 84,63 & 78,33 & 75,84 & 81,18 \\
\hline Classification error (\%) & 15,37 & 21,67 & 24,16 & 18,82 \\
\hline Kappa & 0,809 & 0,730 & 0,699 & 0,767 \\
\hline Recall (\%) & 70,87 & 60,77 & 57,51 & 66,47 \\
\hline Precision (\%) & 76,62 & 66,54 & 62,71 & 71,72 \\
\hline Absolute error & 0,165 & 0,227 & 0,249 & 0,200 \\
\hline Relative error (\%) & 16,50 & 22,72 & 24,86 & 19,96 \\
\hline RMSE & 0,380 & 0,444 & 0,469 & 0,422 \\
\hline
\end{tabular}

Table 8 shows the success rates obtained by using the Tanh, Rectifier, Maxout, and ExpRectifier activation functions after 150 epochs.

Table 8. Success rates of the method after 150 epochs.

\begin{tabular}{|c|c|c|c|c|}
\hline 150 epochs & Tanh & Rectifier & Maxout & ExpRectifier \\
\hline Accuracy (\%) & 82,80 & 80,10 & 75,09 & 81,51 \\
\hline Classification error (\%) & 17,20 & 19,90 & 24,91 & 18,49 \\
\hline Kappa & 0,787 & 0,753 & 0,689 & 0,769 \\
\hline Recall (\%) & 71,25 & 65,13 & 54,30 & 66,07 \\
\hline Precision (\%) & 76,48 & 69,70 & 60,23 & 73,40 \\
\hline Absolute error & 0,175 & 0,206 & 0,254 & 0,194 \\
\hline Relative error (\%) & 17,49 & 20,60 & 25,43 & 19,36 \\
\hline RMSE & 0,395 & 0,425 & 0,478 & 0,414 \\
\hline
\end{tabular}

As seen in the tables above, the highest success was obtained with the tanh activation function. In addition, another parameter that affects success is the epoch number. Since it is understood from the tables that increasing the number of epochs does not increase the success, on the contrary, it decreases the success. From all reasons above, it was understood that the most suitable parameters for the data used in this study were 100 epochs with the tanh activation function.

When the studies in the literature are analyzed, it is seen that there is no study diagnosed for 14 different types of anemia using deep learning methods. Yavuz et al [14] conducted a study on iron deficiency anemia in women. In their study, they decided whether there was iron deficiency using 6 attributes. Although it has a high success rate, only a single disease was diagnosed. In our study, it is a strong aspect that classes are more, and the method used is up to date. Yu et al [32] classified white blood cells using deep learning methods in their study. Xu et al [33], on the other hand, classified the red blood cells using deep learning methods. High successes have also been achieved in the mentioned studies. Although the data used are different from our study in terms of being images. Besides that, their studies are similar to our study because they both diagnose blood diseases, and the methods they use are deep learning methods. Hasani and Hanani [25] used complete blood count parameters similar to our study and they have obtained successful results. In this study, iron deficiency and alpha and beta-thalassemia traits were diagnosed. In our study, 14 different anemia diagnoses were made. Besides, the data we use 
include data such as the patient's age, gender, and other chronic diseases, as well as complete blood count parameters. These data were included in the study because they are the data affecting the doctor's decision for diagnosis. In this respect, our study stands in a different place from other studies.

In the present study, a deep learning-based system was developed to diagnose 14 different types of anemia. The data set was obtained from the actual hospital environment and no intervention, such as increasing or decreasing the number of data, increasing, or decreasing the number of attributes, reduction, integration, imputation, transformation, or discretization, has been made on the incoming patient data. Therefore, since there is no process to change the content and quality of the data, an original dataset is used in this work which is not made any intervention numerically. In addition, the use of real patient data makes this study important. When the dataset is obtained, the data are classified with the deep learning method at the next stage. The deep learning method which is quite popular in the literature has been implemented and significant results have been found. The 10 -fold cross-validation method was used during classification. In this method, the data set is divided into 10 different subsets. When a group is a test set, the remaining 9 groups are set as a training set. In this way, all the combinations are tested so that each of the 10 subsets is once a test set, and as a result, an impressive performance value is found by taking the average of each result.

ROC analysis method was used for performance measurement besides accuracy, classification error, kappa, weighted mean recall and precision, absolute and relative errors, root means squared error methods. An impressive success rate was obtained, as seen in the performance evaluation. It is reasonable that this success will increase when data cleaning and pre-processing are made. In future work, we aim to increase success by using different algorithms of deep learning methods. Also, it is aimed to make various hybrid methods by making additions to these algorithms or using different machine learning methods together, thus, to increase the success ratio. Moreover, by expanding the dataset, hematological diseases other than anemia could also be included in these studies.

\section{Conclusion}

Anemia is a very common disease affecting the quality of life and with the appropriate treatment, the standard of living of the patient will improve. It is obvious that the first step in the treatment is the correct diagnosis. In the present study, the 14 different types of anemia most encountered in Duzce Province were classified by the deep learning method. One of the very important points of this study is, this is the first time with this data and this method is used together. No comparison was made with previous studies because the data used for this study is completely original and with this data, no method has been tried before. Therefore, we believe that any comparison with other studies will not be ethical. For those who want to work in different ways, data can be shared with another permission of the ethics committee which has not been appealed yet.

The strength of the present study was 14 different anemia types are diagnosed like a doctor by the deep learning algorithm. The limitation of this study is that the entire dataset consists of data from individuals with hematologic diseases. In addition, anemia is also a symptom that can accompany many other diseases at the same time. Despite this limitation, it is obvious that the classifier performances obtained are acceptable. This study contributes positively to the literature. Our results showed that it is possible to diagnose like a doctor with the raw data by using the presented method.

Furthermore, in order to improve success, future works should focus on combining different methods and on developing new hybrid methods. Moreover, by expanding the dataset, hematological diseases other than anemia could also be included in our studies.

\section{Acknowledgments}

Funding- This work was supported by the Research Fund of Sakarya University, Turkey, under Project Number: 2015-50-02-010. The funders had no role in study design, data collection and analysis, decision to publish, or preparation of the manuscript. 
Ethical Approval- The data used in this study were taken from the Duzce University Research and Practice Hospital, Turkey, with the approval of the Duzce University Ethics Committee (Decision number 2014/82).

\section{References}

[1] T.Vos, D. Flaxman et al., Years lived with disability (YLDs) for 1160 sequelae of 289 diseases and injuries 1990-2010: A systematic analysis for the Global Burden of Disease Study 2010, The Lancet, 380, 2163-2196, DOI: 10.1016/S0140-6736;12;61729-2, 2012.

[2] Hemoglobin concentrations for the diagnosis of anemia and assessment of severity Vitamin and Mineral Nutrition Information System. Geneva, World Health Organization, Available: http://www.who.int/vmnis/indicators/haemoglobin.pdf (2011).

[3] N.J. Kiassebaum, R. Jasrasaria et al., A systematic analysis of global anemia burden from 1990 to 2010. Blood, 123, 615-624, DOI: 10.1182/blood-2013-06-508325, 2014.

[4] J.R. Beck, J.R. Bell, F.Hirai, J.J. Simmons, H.C. Jr. Lyon, Computer-Based Exercises in Cardiac Diagnosis (PlanAlyzer), Proc. Annu. Symp. Computer Applications in Medical Care, Nov 9, 403-408, PMCID: PMC2245328, 1988.

[5] J.R. Beck, J.F. O’Donnell, F. Hirai, J.J. Simmons, J.C. Healy, H. C. Jr. Lyon, Computer-based exercises in anemia diagnosis (PlanAlyzer), Methods Inf. Med., 28:4, 364-369, PMID:2695787, 1989.

[6] H. C. Jr. Lyon, J.R. Bell, J.F. O’Donnell, F. Hirai, J.C. Healy, J.R. Beck, The PlanAlyzer Cases for Teaching Clinical Reasoning: A Demonstration of the Cases, Discussion of the Research \& Development Process, Lessons Learned and Strategies for Introducing Computer-Based Programs into Medical School Courses as a Vehicle for Curriculum Reform, Proc. Annu. Symp. Computer Applications in Medical Care, PMCID: PMC2850769, 1993.

[7] M. Lipkin, Correlation of Data with a Digital Computer in the Differential Diagnosis of Hematological Diseases, IRE Transactions on Medical electronics, 243-246, 1960.

[8] R. L. Engle, B. J. Flehinger, S. Allen, R. Friedman, M. Lipkin, B. J. Davis, L. L. Leveridge, HEME: A Computer Aid to Diagnosis of Hematologic Disease, Bulletin of the New York Academy of Medicine, 52:5, 584-600, 1976.

[9] I. Azarkhish, M.R. Raoufy, S. Gharibzadeh, Artificial Intelligence Models for Predicting Iron Deficiency Anemia and Iron Serum Level Based on Accessible Laboratory Data, J Med Syst, 36, 2057-2061, DOI: 10.1007/s10916-011-9668-3, 2012.

[10] Z. Y1lmaz, M.R. Bozkurt, Determination of Women Iron Deficiency Anemia Using Neural Networks, J Med Syst, 36, 2941-2945, DOI: 10.1007/s10916-011-9772-4, 2012.

[11] A. Y1lmaz, M. Dağlı, N. Allahverdi, A Fuzzy Expert System Design for Iron Deficiency Anemia, IEEE 7th International Conference on Application of Information and Communication Technologies (AICT), Baku, Azerbaijan, DOI: 10.1109/ICAICT.2013.6722707, 2013.

[12] W. Reinisch, M. Staun, S Bhandari., M. Munoz, State of the iron: How to diagnose and efficiently treat iron deficiency anemia in inflammatory bowel disease, Journal of Crohn's and Colitis, 7, 429-440, 2013.

[13] Ş. Doğan, I. Türkoğlu, Iron-Deficiency Anemia Detection from Hematology Parameters by Using Decision Trees, International Journal of Science \& Technology, 3:1, 85-92, 2008.

[14] B.C. Yavuz, T. Karagül Yıldız, N. Yurtay, Z.Y1lmaz, Comparison of K Nearest Neighbors and Regression Tree Classifiers Used with Clonal Selection Algorithm to Diagnose Hematological Diseases, AJIT-e: Online Academic Journal of Information Technology, 5-16, DOI: 10.5824/1309-1581.2014.3.001. x, 2014.

[15] S. A. Sanap, M. Nagori, V. Kshirsagar, Classification of Anemia Using Data Mining Techniques, Presented in SEMCCO 2011, Berlin Heidelberg, Part II, 2011.

[16] N. Amin, A. Habib, Comparison of Different Classification Techniques Using WEKA for Hematological Data, Am J Eng Res, 4:3, 55-61, e-ISSN: 2320- 0847, 2015.

[17] I. Yılmaz, Demir eksikliği anemisi ile beta talasemi minörün ayırıcı tanısında eritrosit indekslerin rolü, Expertise Thesis, Dept. of Internal Medicine, Faculty of Medicine, Pamukkale University, Denizli, Turkey, 2010. 
[18] E. Urrechaga, U. Aguirre, S. Izquierdo, Differential Diagnosis of Microcytic Anemia, Anemia, DOI: 10.1155/2013/457834, 2013.

[19] M. K. Jamei, K. M. Talarposhti, Discrimination between Iron Deficiency Anaemia (IDA) and $\beta$ - Thalassemia Trait ( $\beta$-TT) Based on Pattern-Based Input Selection Artificial Neural Network (PBIS- ANN), J Adv Comp Res, 7: 4, 55-66, pISSN: 2345-606x, eISSN: 2345-6078, 2016.

[20] R. Kishore, K.P. Rao, G.R.S. Murthy, Performance Evaluation of Entropy and Gini using Threaded and Non-Threaded ID3 on Anaemia Dataset, Presented at Fifth International Conference on Communication Systems and Network Technologies, IEEE, 2015.

[21] M. F. Shaik, M. Subashini, Anemia Diagnosis by Fuzzy Logic Using LabVIEW, Presented at IEEE International Conference on Intelligent Computing and Control (I2C2), DOI: 10.1109/I2C2.2017.8321790, 2017.

[22] P. T. Dalvi, N. Vernekar, Anemia Detection using Ensemble Learning Techniques and Statistical Models, Presented at IEEE International Conference on Recent Trends in Electronics Information Communication Technology, May 20-21, 2016.

[23] S. Belginova, I. Uvaliyeva, A. Ismukhamedova, Decision Support System for Diagnosing Anemia, Presented at 4th International Conference on Computer and Technology Applications, DOI: 10.1109/CATA.2018.8398684, 2018.

[24] G. Dimauro, D. Caivano, F. Girardi, A New Method and a Non-Invasive Device to Estimate Anemia Based on Digital Images of the Conjunctiva, IEEE Access-Special Section on HumanCentered Smart Systems and Technologies, DOI: 10.1109/ACCESS.2018.2867110, 2018.

[25] M. Hasani, A. Hanani, Automated Diagnosis of Iron Deficiency Anemia and Thalassemia by Data Mining Techniques, International Journal of Computer Science and Network Security, 17:4, 326-331, 2017.

[26] G. Gunčar, M. Kukar, M. Notar, M. Brvar, P. Černelč, M. Notar, M. Notar, An application of machine learning to haematological diagnosis, Scientific Reports, 8:411, 1-12, DOI:10.1038/s41598-017-18564-8, 2018.

[27] H. Ayyıldız, S. A. Tuncer, Determination of the effect of red blood cell parameters in the discrimination of iron deficiency anemia and beta thalassemia via Neighborhood Component Analysis Feature Selection-Based machine learning, Chemometrics and Intelligent Laboratory Systems, 196, 1-8, DOI: https://doi.org/10.1016/j.chemolab.2019.103886, 2020.

[28] J. Schmidhuber, Deep learning in neural networks: An overview, Neural Networks, 61, 85-117. DOI: 10.1016/j.neunet.2014.09.003, 2015.

[29] W. Liu, Z. Wang, X. Liu, N. Zeng, Y. Liu, F. E. Alsaadi, A survey of deep neural network architecture and their applications, Neurocomputing, 234, 11-26, DOI: 10.1016/j.neucom.2016.12.038, 2017.

[30] G. Litjens et al., A survey on deep learning in medical image analysis, Medical Image Analysis, 42, 60-88, DOI: 10.1016/j.media.2017.07.005, 2017.

[31] R. Miotto, L. Li, B. A. Kidd, J. T. Dudley, Deep Patient: An Unsupervised Representation to Predict the Future of Patients from the Electronic Health Records, Scientific Reports, 6:26094,110. DOI: 10.1038/srep26094, 2016.

[32] W. Yu, J. Chang, C. Yang, L. Zhang, H. Shen, Y. Xia, J. Sha, Automatic Classification of Leukocytes Using Deep Neural Network, IEEE 12th International Conference on ASIC, DOI: 10.1109/ASICON.2017.8252657, 2017.

[33] M. Xu, D.P. Papageorgiou, S.Z. Abidi, M. Dao, H. Zhao, G.E. Karniadakis, A deep convolutional neural network for classification of red blood cells in sickle cell anemia, PLoS Computational Biology, 13:10, DOI: https://doi.org/10.1371/journal.pcbi.1005746, 2017.

[34] K. Kimura, Y. Tabe, T. Ai, I. Takehara, H. Fukuda, H. Takahashi, T. Naito, N. Komatsu, K. Uchihashi, A. Ohsaka, A novel automated image analysis system using deep convolutional neural networks can assist to differentiate MDS and AA, Scientific Reports, 9, 1-9, DOI: https://doi.org/10.1038/s41598-019-49942-z, 2019.

[35] F. K. Alsheref, W. H. Gomaa, Blood Diseases Detection using Classical Machine Learning Algorithms, International Journal of Advanced Computer Science and Applications, 10:7, 7781, 2019.

[36] B. Çil, H. Ayyıldız, T. Tuncer, Discrimination of $\beta$-thalassemia and iron deficiency anemia 
through extreme learning machine and regularized extreme learning machine-based decision support system, Medical Hypotheses, 138, 1-6, DOI: https://doi.org/10.1016/j.mehy.2020.109611, 2020.

[37] N. Varghese, Machine Learning Techniques for the Classification of Blood Cells and Prediction of Diseases, International Journal of Computer Science Engineering, 9:1, 66-75, 2020.

[38] L. Alzubaidi, M. A. Fadhel, O. Al - \$hðhamm, Y. Duan, Deep Learning Models for Classification of Red Blood Cells in Microscopy Images to Aid in Sickle Cell Anemia Diagnosis, Electronics, 9:427, 1-20, DOI:10.3390/electronics9030427, 2020.

[39] S. Devunooru, A. Alsadoon, P. W. C. Chandana, A. Beg, Deep learning neural networks for medical image segmentation of brain tumours for diagnosis: a recent review and taxonomy, Journal of Ambient Intelligence and Humanized Computing (2021) 12:455-483, https://doi.org/10.1007/s12652-020-01998-w

[40] E. Goceri, Deep learning based classification of facial dermatological disorders, Computers in Biology and Medicine, Volume 128, January 2021, 104118, https://doi.org/10.1016/j.compbiomed.2020.104118

[41] S. KILICARSLAN, M. CELIK, SS. SAHIN, Hybrid models based on genetic algorithm and deep learning algorithms for nutritional Anemia disease classification, Biomedical Signal Processing and Control, Volume 63, January 2021, 102231, https://doi.org/10.1016/j.bspc.2020.102231

[42] S. Yeruva, M. S. Varalakshmi, B. P. Gowtham, Y. H. Chandana, PESN. K. Prasad, Identification of Sickle Cell Anemia Using Deep Neural Networks, Emerging Science Journal (ISSN: 26109182) Vol. 5, No. 2, April, 2021, http://dx.doi.org/10.28991/esj-2021-01270

[43] A. Gupta, Anjum, S. Gupta, R. Katarya, InstaCovNet-19: A deep learning classification model for the detection of COVID-19 patients using Chest X-ray, Applied Soft Computing, Volume 99, February 2021, 106859, https://doi.org/10.1016/j.asoc.2020.106859

[44] J. Venugopalan, L. Tong, H. R. Hassanzadeh, M. D. Wang, Multimodal deep learning models for early detection ofAlzheimer's disease stage, Scientific Reports, (2021) vol. 11, article number. 3254, https://doi.org/10.1038/s41598-020-74399-w

[45] Ü. Atila, M. Uçar, K. Akyol, E. Uçar, Plant leaf disease classification using EfficientNet deep learning model, Ecological Informatics, Volume 61, March 2021, 101182, https://doi.org/10.1016/j.ecoinf.2020.101182

[46] Haematology in Clinical Practice, Part 1: Erythrocyte Disorders, Chapter 2. Clinical Approach to Anemia, 5th ed., Güneş Medical Bookstore, ISBN: 978-975-277-404-9, 2012.

[47] B. Onec, Personal Lecture Notes of Birgül Öneç, Duzce University, Medical School, Department of Internal Medicine Sciences, 2017.

[48] R. S. Hillman, K. A. Ault, M. Leporrier, H. M. Rinder, Haematology in Clinical Practice. Part 1: Red Blood Cell Disorders, Chapter 2. Clinical Approach to Anemia, 5th ed., Istanbul, Turkey: Güneş Medical Bookstore, ISBN: 978-975-277-404-9, 2012.

[49] Guide to diagnosis and treatment of Erythrocyte Diseases and Hemoglobin disorders, Turkish Hematology Association, Version1-July2011, Available: www.thd.org.tr, 2011.

[50] R. Hoffman, E.J. Benz, L.E. Silberstein, H.E. Heslop, Weitz J.I., Anastasi J., Hematology: Basic Principles and Practice, 6th edition, ISBN: 978-1-4377-2928-3, Elsevier, 2013.

[51] A. E. Hassanien, E. T. Al-Shammari, N. Ghali, Computational Intelligence Techniques in Bioinformatics, Computational Biology and Chemistry, DOI: 10.1016/j.compbiolchem. 2013.04.007, 2013.

[52] E. Öztemel, Artificial Neural Networks, Papatya Press, Istanbul, Turkey, 2006. ISBN: 975-6797-39-8.

[53] Rapidminer $\quad$ Tutuorial, $2017 . \quad$ [Online]. Available: https://docs.rapidminer.com/studio/operators/modeling/predictive/neural_nets/deep_learning.ht ml [Accessed: 30.10.2017].

[54] T. Fawcett, An introduction to ROC analysis, Pattern Recognition Letters, 27, 861-874, 2006.

[55] F.Ramzan, M.U.G.Khan, A.Rehmat, S.Iqbal, T.Saba, A.Rehman, Z.Mehmood, A deep learning approach for automated diagnosis and multi-class classification of Alzheimer's disease stages using resting-state fMRI and residual neural networks, Journal of Medical Systems vol.44(37), 2020. 